\title{
CALCULATION METHOD OF KINETIC CONSTANTS FOR THE MATHEMATICAL MODEL PEAT PYROLYSIS
}

\author{
Tatyana Plakhova, Alexsandr Subbotin
}

National Research Tomsk Polytechnic University, 30, Lenin Avenue, 634050 Tomsk, Russia

\begin{abstract}
Relevance of the work is related to necessity to simplify the calculation of kinetic constants for the mathematical model peat pyrolysis. Execute transformations of formula Arrhenius law. Degree of conversion is expressed in terms mass changes of sample. The obtained formulas help to calculate the kinetic constants for any type of solid organic fuels
\end{abstract}

\section{Introduction}

One of the most popular renewable energy sources in the energy balance of the Tomsk region is low-grade fuel, which include peat. For convenient and high-quality burning is proposed to use low-temperature pyrolysis promising technology [1].

There is a mathematical model for the pyrolysis process, which helps to calculate the thermal effect from solid fuels. For each type of solid fuel in this mathematical model is necessary to substitute the individual kinetic constants - pre-exponential factor $\mathrm{k}_{0}$ and activation energy $E, \mathrm{~kJ} / \mathrm{mol}$.

In this paper the simplified method of calculation of kinetic constants using the experimental data of the fuel mass changes during thermal exposure is shown.

\section{Object of study}

Generally all reactions of decomposition and combustion of solid fuels are described by first order differential equations [6]. Arrhenius law for pyrolysis describing was used [2]:

$$
\frac{d \alpha}{d t}=(1-\alpha) k_{0} \cdot \exp \left(-\frac{E}{R T}\right)
$$

where $\quad \alpha$-degree of conversion;

$\mathrm{k}_{0}$ - pre-exponential factor (determined by experiment); 
$\mathrm{E}$ - activation energy (determined by experiment); $\mathrm{kJ} / \mathrm{mol}$.

$\mathrm{R}$ - universal gas constant $=8,314 \mathrm{~J} / \mathrm{mol} \cdot K$;

$\mathrm{T}$ - sample temperature, $K$;

To graph dependence of mass changes on the time applies thermal analysis (DTA, TGA DTG) which is carried out by setting the thermal analyzer SDT Q600. For example, used a sample of peat deposits Kandinsky, which was heated at a rate of $5{ }^{\circ} \mathrm{C} / \mathrm{min}$. On the resulting curve selected ranges start and end of the main combustion reaction and the corresponding operating points for mass and temperature.

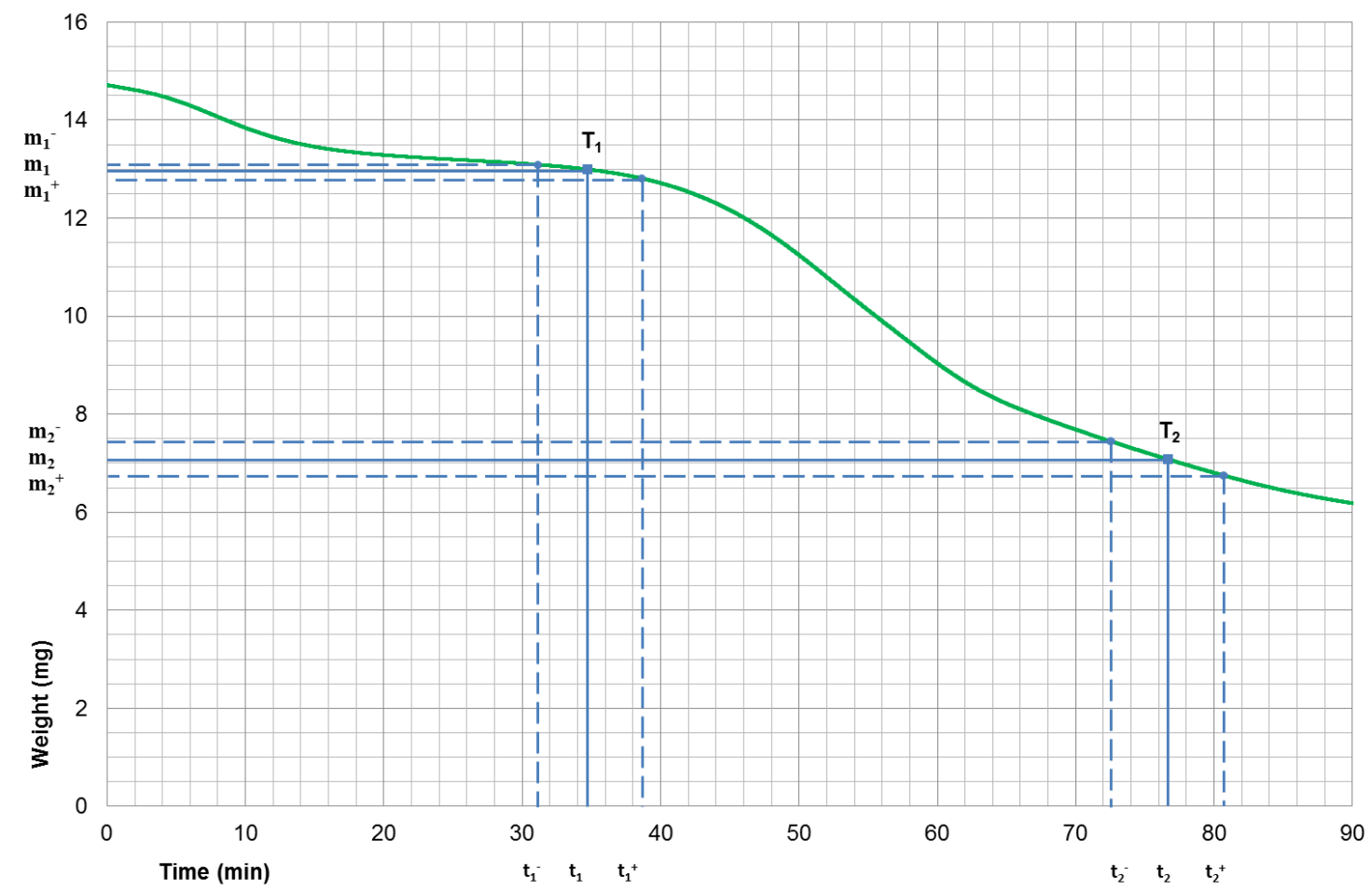

Figure 1. Mass changing from time curve for peat sample with heating rate $5^{\circ} \mathrm{C} / \mathrm{min}$.

Experimentally determined mass changing allowed switching to mass in (1) from conversion level:

$$
\alpha=\frac{m_{\text {initial }}-m}{m_{\text {initial }}-m_{\text {final }}} ;
$$




$$
\begin{gathered}
-(1-\alpha)=-\left(1-\frac{m_{\text {initial }}-m}{m_{\text {initial }}-m_{\text {final }}}\right)= \\
=\left(\frac{-m_{\text {initial }}+m_{\text {final }}+m_{\text {initial }}-m}{m_{\text {initial }}-m_{\text {final }}}\right)=\frac{m_{\text {final }}-m}{m_{\text {initial }}-m_{\text {final }}} \\
\frac{1}{m_{\text {initial }}-m_{\text {final }}} \cdot \frac{\mathrm{dm}}{d t}=\frac{m_{\text {final }}-m}{m_{\text {initial }}-m_{\text {final }}} \cdot \mathrm{k}_{0} \exp \left(-\frac{E}{R T}\right) \\
\frac{\mathrm{dm}}{d t}=\left(m_{\text {final }}-m\right) k_{0} \exp \left(-\frac{E}{R T}\right)
\end{gathered}
$$

Equations for two points with temperatures $T_{1}$ and $T_{2}$ and masses $m_{1}$ and $m_{2}$ were obtained. As a result the equations system with two unknown elements was produced:

$$
\begin{aligned}
& \mathrm{k}_{0}=\frac{\mathrm{dm}_{1}}{\mathrm{dt}} \cdot \frac{1}{\mathrm{~m}_{\text {final }}-\mathrm{m}_{1}} \cdot \exp \left(\frac{\mathrm{E}}{\mathrm{RT}_{1}}\right) ; \\
& \mathrm{k}_{0}=\frac{\mathrm{dm}_{2}}{\mathrm{dt}} \cdot \frac{1}{\mathrm{~m}_{\text {final }}-\mathrm{m}_{2}} \cdot \exp \left(\frac{\mathrm{E}}{\mathrm{RT}_{2}}\right) .
\end{aligned}
$$

Equations were solved regarding to $\mathrm{k}_{0}$. Right parts of (4) and (5) were equaled:

$$
\frac{\mathrm{dm}_{1}}{\mathrm{dt}} \cdot \frac{1}{\mathrm{~m}_{\text {final }}-\mathrm{m}_{1}} \cdot \exp \left(\frac{\mathrm{E}}{\mathrm{RT}_{1}}\right)=\frac{\mathrm{dm}_{2}}{\mathrm{dt}} \cdot \frac{1}{\mathrm{~m}_{\text {final }}-\mathrm{m}_{2}} \cdot \exp \left(\frac{\mathrm{E}}{\mathrm{RT} \mathrm{T}_{2}}\right) .
$$

Activation energy calculation equation was found as:

$$
\begin{aligned}
& \exp \left(\frac{E}{\mathrm{RT}_{1}}-\frac{\mathrm{E}}{\mathrm{RT}_{2}}\right)=\frac{\frac{\mathrm{dm}}{\mathrm{dt}} \cdot \frac{1}{\mathrm{~m}_{\text {final }}-\mathrm{m}_{2}}}{\frac{\mathrm{dm}}{\mathrm{dt}} \cdot \frac{1}{\mathrm{~m}_{\text {final }}-\mathrm{m}_{1}}} ; \\
& \exp \mathrm{E}\left(\frac{1}{\mathrm{RT}_{1}}-\frac{1}{\mathrm{RT}_{2}}\right)=\exp \cdot \frac{\mathrm{E}\left(\mathrm{T}_{2}-\mathrm{T}_{1}\right)}{\mathrm{RT}_{1} \cdot \mathrm{T}_{2}} ;
\end{aligned}
$$




$$
\begin{aligned}
& \exp \frac{\mathrm{E}\left(\mathrm{T}_{2}-\mathrm{T}_{1}\right)}{\mathrm{RT}_{1} \cdot \mathrm{T}_{2}}=\frac{\dot{\mathrm{m}}_{2}\left(\mathrm{~m}_{\text {final }}-\mathrm{m}_{1}\right)}{\left(\mathrm{m}_{\text {final }}-\mathrm{m}_{2}\right) \dot{\mathrm{m}}_{1}} ; \\
& \frac{\mathrm{E}\left(\mathrm{T}_{2}-\mathrm{T}_{1}\right)}{\mathrm{RT}_{1} \cdot \mathrm{T}_{2}}=\ln \frac{\dot{\mathrm{m}}_{2}\left(\mathrm{~m}_{\text {final }}-\mathrm{m}_{1}\right)}{\dot{\mathrm{m}}_{1}\left(\mathrm{~m}_{\text {final }}-\mathrm{m}_{2}\right)} ; \\
& \mathrm{E}=\frac{\mathrm{RT}_{1} \mathrm{~T}_{2}}{\mathrm{~T}_{2}-\mathrm{T}_{1}} \cdot \ln \frac{\dot{\mathrm{m}}_{2}\left(\mathrm{~m}_{\text {final }}-\mathrm{m}_{1}\right)}{\dot{\mathrm{m}}_{1}\left(\mathrm{~m}_{\text {final }}-\mathrm{m}_{2}\right)}
\end{aligned}
$$

Pre-exponential factor was calculated using (4) and (5):

$$
\mathrm{k}_{0}=\frac{\mathrm{m}_{\mathrm{n}}}{\mathrm{m}_{\text {final }}-\mathrm{m}_{\mathrm{n}}} \cdot \exp \frac{\mathrm{E}}{\mathrm{RT}_{\mathrm{n}}},
$$

where $n$-point on the curve of mass changing from time.

\section{Conclusion}

Shown an improved method of calculation allows more quickly determine the kinetic constants for different types of peat on the basis of experimental studies in the absence of the necessary reference data.

The reported study was partially supported by the Ministry of education and science of The Russian Federation (Government Order No. 13.948.2014/K).

\section{References}

[1] R.B. Tabakaev, A.V. Kazakov and A.S. Zavorin, "Prospects of using low-grade fuels of Tomsk region for the thermal technology use," Bulletin of Tomsk Polytechnic University, vol. 4 (323), pp. 41-46, 2013.

[2] V.V. Pomerantsev, Fundamentals of Practical Combustion Theory, L.: Energoatomizdat, 1986, p. 312. [In Russian]. 